\title{
Efficient C-Planarity Testing for Embedded Flat Clustered Graphs with Small Faces ${ }^{\star}$
}

\author{
Giuseppe Di Battista and Fabrizio Frati \\ Dipartimento di Informatica e Automazione - Università di Roma Tre
}

\begin{abstract}
Let $C$ be a clustered graph and suppose that the planar embedding of its underlying graph is fixed. Is testing the $c$-planarity of $C$ easier than in the variable embedding setting? In this paper we give a first contribution towards answering the above question. Namely, we characterize $c$-planar embedded flat clustered graphs with at most five vertices per face and give an efficient testing algorithm for such graphs. The results are based on a more general methodology that shades new light on the $c$-planarity testing problem.
\end{abstract}

\section{Introduction}

Determining the computational complexity of the $c$-planarity testing for clustered graphs is one of the main Graph Drawing challenges. However, despite all the research efforts spent, only for restricted families of clustered graphs polynomial-time testing algorithms have been found, and the general problem is open.

A brief survey on the problem of testing the $c$-planarity of clustered graphs can be found in [2]. The classes of clustered graphs for which the problem is known to be polynomial-time solvable are the following. c-Connected clustered graphs, in which each cluster induces a connected subgraph of the underlying graph; the first algorithm for this class has been presented in [7]. Completely connected clustered graphs, that are $c$-connected clustered graphs such that the complement of the subgraph induced by each cluster is connected; an elegant characterization for this class is shown in [1]. Almost connected clustered graphs, in which either all nodes corresponding to non-connected clusters are in the same path in the cluster hierarchy, or for each non-connected cluster its parent and all its siblings are connected [9]. Extrovert clustered graphs, a generalization of $c$-connected clustered graphs with special restrictions on the cluster hierarchy [8]. Cycles of clusters, in which the hierarchy is flat, the underlying graph is a simple cycle, and the clusters are arranged in a cycle [3]. The clustering hierarchy is flat if all clusters, but for the root, are at the same level. Clustered cycles, that are clustered graphs in which the hierarchy is flat, the underlying graph is a simple cycle, and the clusters are arranged into an embedded plane graph [4].

Let $C$ be a clustered graph. Suppose that a planar embedding of its underlying graph is fixed. Is testing the $c$-planarity of $C$ easier than in the variable embedding setting? This question is motivated by the existence of many NP-hard Graph Drawing problems

\footnotetext{
* Work partially supported by MUR under Project MAINSTREAM Algorithms for Massive Information Structures and Data Streams.
} 
on planar graphs that become polynomial-time solvable if the embedding is fixed. Testing if a graph admits an orthogonal planar drawing with at most $k$ bends or if a graph admits an upward planar drawing are examples of such problems.

In this paper we give a first contribution towards answering the above question. Namely, we characterize $c$-planar embedded flat clustered graphs with at most five vertices per face and give an efficient testing algorithm for such graphs. Our approach is to look for an augmentation of the embedded underlying graph with extra edges such that the resulting graph is $c$-connected and $c$-planar. We call candidate saturating edges those edges that are candidates for the augmentation. Two of such edges have a conflict if using both of them in the augmentation causes a crossing. We present a characterization and an efficient $c$-planarity testing algorithm for single-conflict embedded flat clustered graphs, that are embedded clustered graphs such that (i) the cluster hierarchy is flat and (ii) each candidate saturating edge has a conflict with at most one other candidate saturating edge. Characterization and algorithm for clustered graphs with at most five vertices per face are a consequence of such a more general result.

The paper is organized as follows: In Section 2 we give preliminaries. In Section 3 we characterize $c$-planar single-conflict embedded flat clustered graphs and $c$-planar embedded flat clustered graphs with at most five vertices per face. In Section 4 we present a linear time and space $c$-planarity test. Section 5 contains conclusions and open problems. Because of space limits some proofs are in the full version of the paper [6].

\section{Preliminaries}

A graph $G$ is vertex (edge) $k$-connected if the removal of any $k-1$ vertices (edges) leaves $G$ connected. A separating edge is an edge whose removal disconnects $G$.

A drawing of a graph is a mapping of each vertex to a distinct point of the plane and of each edge to a Jordan curve between the endpoints of the edge. A planar drawing is such that no two edges intersect except, possibly, at common endpoints. A planar drawing of a graph determines a circular ordering of the edges incident to each vertex. Two drawings of the same graph are equivalent if they determine the same circular orderings around each vertex. A planar embedding is an equivalence class of planar drawings. A planar drawing partitions the plane into topologically connected regions, called faces. The unbounded face is the outer face. Two planar drawings with the same planar embedding have the same faces. However, such drawings could still differ for their outer face. The dual graph $D$ of a planar embedded graph $G$ is the graph with a vertex for each face of $G$ and with an edge $e(D)$ between two vertices if the corresponding faces share an edge $e(G)$; edge $e(D)$ is dual to edge $e(G)$. In the following we will deal both with biconnected (that is vertex 2-connected) and with simply connected (that is vertex 1 -connected) embedded planar graphs. In the former case, the "number of vertices in a face" is trivially defined as the number of vertices incident to the face, while in the latter one is meant to be the number of occurrences of vertices in the border of the face.

A clustered graph is a pair $C(G, T)$, where $G$ is a planar graph and $T$ is a rooted tree whose leaves are the vertices of $G$. Graph $G$ and tree $T$ are called underlying graph and inclusion tree, respectively. Each internal node $\mu$ of $T$ corresponds to the subset $V(\mu)$ (called cluster) of the vertices of $G$ that are leaves of the subtree of $T$ rooted at $\mu ; G(\mu)$ 


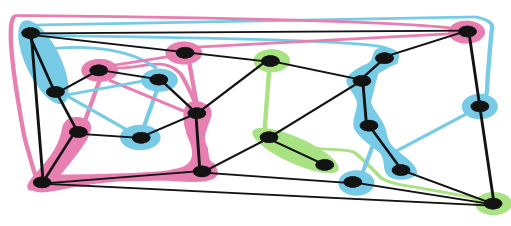

(a)

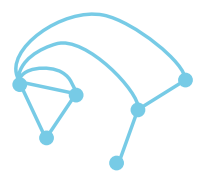

(b)

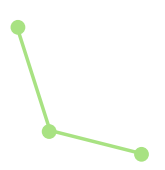

(c)

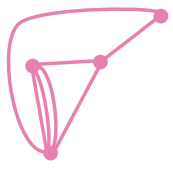

(d)

Fig. 1. (a) An embedded flat clustered graph $C$ and its candidate saturating edges. Different clusters have different colors. (b)-(c)-(d) Multigraphs $\mathcal{G}_{i}$ for $C$.

denotes the subgraph of $G$ induced by the vertices in $V(\mu)$. If each cluster induces a connected subgraph of $G$, then $C$ is c-connected, otherwise $C$ is non-c-connected. An embedded clustered graph is a clustered graph such that $G$ is connected and the planar embedding of the underlying graph of $C$ is fixed. A flat clustered graph is such that the number of nodes in any path from the root to a leaf of $T$ is three. When referring to a flat clustered graph, given a vertex $v$ of the underlying graph we say that the cluster of $v$ is its parent in $T$. Also, we call clusters only the children of the root.

A drawing of a clustered graph $C(G, T)$ consists of a drawing of $G$ and of a representation of each node $\mu$ of $T$ as a simple closed region $R(\mu)$ such that: (i) $R(\mu)$ contains the drawing of $G(\mu)$; (ii) $R(\mu)$ contains a region $R(\nu)$ iff $\nu$ is a descendant of $\mu$ in $T$; and (iii) the borders of any two regions don't intersect. Consider an edge $e$ and a node $\mu$ of $T$. If $e$ crosses the boundary of $R(\mu)$ more than once, we say that edge $e$ and region $R(\mu)$ have an edge-region crossing. A drawing of a clustered graph is $c$-planar if it does not have edge crossings or edge-region crossings. A clustered graph is $c$-planar if it admits a $c$-planar drawing. An embedded clustered graph is $c$-planar if it admits a $c$-planar drawing in which the embedding of $G$ is preserved.

Consider an embedded flat clustered graph $C(G, T)$. For each face $f$ of $G$ a set of candidate saturating edges is defined as follows: Let $O$ be the clockwise circular order of the vertices on the border of $f$. Subdivide such vertices into subsets such that each subset $V_{i}$ contains a maximal sequence of consecutive vertices in $O$ belonging to the same cluster. Introduce a candidate saturating edge for each $V_{i} \neq V_{j}$ such that (i) $V_{i}$ and $V_{j}$ contain vertices of the same cluster $\mu_{k}$ and (ii) $V_{i}$ and $V_{j}$ are in different connected components of $G\left(\mu_{k}\right)$. Candidate saturating edges are edges that can be added to the clustered graph to make it $c$-connected (see Fig. 1). For a cluster $\mu_{i}$ of $T$ we define $\mathcal{G}_{i}$ as the embedded multigraph whose vertices are the connected components of $G\left(\mu_{i}\right)$ and whose edges are the candidate saturating edges. The embedding of $\mathcal{G}_{i}$ is given by the order of the faces around the vertices of $G$. Observe that $\mathcal{G}_{i}$ does not have self-loops and is, in general, non-planar. However, possible crossings are only between edges introduced in the same face of $G$. Two candidate saturating edges $e_{1}$ and $e_{2}$, joining connected components $G_{1}\left(\mu_{i}\right)$ and $G_{2}\left(\mu_{i}\right)$ of $G\left(\mu_{i}\right)$, and $G_{1}\left(\mu_{j}\right)$ and $G_{2}\left(\mu_{j}\right)$ of $G\left(\mu_{j}\right)$, respectively, with $\mu_{i} \neq \mu_{j}$ and with $e_{1}$ and $e_{2}$ in the same face $f$ of $G$, have a conflict if $G_{1}\left(\mu_{i}\right), G_{1}\left(\mu_{j}\right), G_{2}\left(\mu_{i}\right)$, and $G_{2}\left(\mu_{j}\right)$ appear in this order around the border of $f$. Informally, two candidate saturating edges have a conflict if adding both of them to the clustered graph causes a crossing. The following theorem shows the role of candidate saturating edges for the $c$-planarity of a flat embedded clustered graph. Even if not explicitly stated, Theorem 1 has been used in [3]. 
Theorem 1. An embedded flat clustered graph $C(G, T)$ is c-planar if and only if: (1) $G$ is planar; (2) there exists a face $f$ in $G$ such that when $f$ is chosen as outer face for $G$ no cycle composed by vertices of the same cluster encloses a vertex of a different cluster; (3) it is possible to augment $G$ to a graph $G^{\prime}$ by adding a subset of the candidate saturating edges of $C$ so that no two added edges have a conflict and each cluster induces in $G^{\prime}$ exactly one connected component.

Hence, given an embedded flat clustered graph $C(G, T)$, if Conditions 1 and 2 are satisfied by $G$, the problem of testing the $c$-planarity of $C$ can be restated as the problem of testing if it is possible to select from multigraphs $\mathcal{G}_{i}$ a set of candidate saturating edges to enforce Condition 3. If such a set exists, we call it a saturator of $C$.

Lemma 1. An embedded flat clustered graph $C(G, T)$ admits a saturator if and only if it admits an acyclic saturator.

Hence, testing the $c$-planarity of an embedded flat clustered graph satisfying Conditions 1 and 2 of Theorem 1 is the same of testing if there exists a spanning tree of each $\mathcal{G}_{i}$ where no two edges in different spanning trees have a conflict.

\section{A Characterization}

We restrict ourselves to embedded flat clustered graphs in which each candidate saturating edge has a conflict with at most one other candidate saturating edge. We call single-conflict an embedded flat clustered graph satisfying such a property. Consider a single-conflict embedded flat clustered graph $C(G, T)$ and the multigraph $\mathcal{G}_{i}$ for each cluster $\mu_{i}$ in $T$. We have the following structural lemma.

Lemma 2. If a graph $\mathcal{G}_{i}$ contains two crossing edges $e_{1}$ and $e_{2}$, then $e_{1}$ and $e_{2}$ have no conflict with edges of other multigraphs.

By Lemma 3 we can assume that in the interesting cases the $\mathcal{G}_{i}$ 's are connected.

Lemma 3. If there exists a $\mathcal{G}_{i}$ that is not connected, then $C$ is not c-planar.

There are edges in the $\mathcal{G}_{i}$ 's that must be used in any saturator of $C$ and edges that are not used in any saturator. Further, there are edges that can be supposed to belong to a saturator without altering the possibility to have one. Roughly speaking, such edges do not belong to the "core" of the problem. Hence, in the following we simplify the $\mathcal{G}_{i}$ 's with an algorithm that either returns that $C$ is not $c$-planar or returns a structure where there are no trivial choices. For this purpose, we define two operations on $\mathcal{G}_{i}$.

The operation of removing an edge $e$ from $\mathcal{G}_{i}$ corresponds to the choice of not using $e$ in the saturator of $C$. Notice that, when an edge $e$ is removed from $\mathcal{G}_{i}$, an edge of $\mathcal{G}_{j}$, with $i \neq j$, that possibly had a conflict with $e$ does not have a conflict any longer.

The operation of collapsing an edge $e$ with end-vertices $u$ and $v$ in $\mathcal{G}_{i}$ corresponds to the choice of using $e$ in the saturator of $C$. It consists of: (i) deleting vertices $u$ and $v$, (ii) removing from $\mathcal{G}_{i}$ all edges between $u$ and $v$, and (iii) inserting in $\mathcal{G}_{i}$ a new vertex whose incident edges are those of $u$ and $v$. The embedding of $\mathcal{G}_{i}$ is preserved. The 
collapsing operation "preserves" the conflicts. Namely, let $e_{i}$ be an edge of $\mathcal{G}_{i}$ incident to $u$ or in $v$ but not in both. Suppose that $e_{i}$ has a conflict (has not a conflict) with an edge $e_{j}$ of $\mathcal{G}_{j}$, with $i \neq j$. After collapsing edge $e$ in a new vertex $w$ the edge incident to $w$ corresponding to $e_{i}$ has a conflict (resp. has not a conflict) with $e_{j}$.

The algorithm is as follows. Repeatedly modify the $\mathcal{G}_{i}$ 's by applying one of the following simplifications. From now on, $\mathcal{G}_{i}$ denotes the multigraph obtained from the starting $\mathcal{G}_{i}$ after some simplifications have been performed. Simplification 1: If there exists an edge $e$ of a $\mathcal{G}_{i}$ that has no conflict, then collapse $e$ in $\mathcal{G}_{i}$. Simplification 2: If there exist a separating edge $e_{i}$ and a non-separating edge $e_{j}$ that are in $\mathcal{G}_{i}$ and $\mathcal{G}_{j}$, respectively, and that conflict each other, then collapse $e_{i}$ in $\mathcal{G}_{i}$ and remove $e_{j}$ from $\mathcal{G}_{j}$. Simplification 3: If there exist two separating edges $e_{i}$ and $e_{j}$ that are in $\mathcal{G}_{i}$ and $\mathcal{G}_{j}$, respectively, and that conflict each other, then stop because $C$ is not $c$-planar.

If the algorithm does not stop for non-c-planarity, we call the final $\mathcal{G}_{i}$ candidate saturating graph for cluster $\mu_{i}$ and we denote it by $\mathcal{G}_{i}^{*}$. Also, we say that $\mu_{i}$ admits a candidate saturating graph. The following properties can be easily proved.

Property 1. None of Simplifications 1, 2, and 3 could disconnect any $\mathcal{G}_{i}$.

Property 2. The subgraphs induced by the collapsed edges are acyclic.

Property 3. Candidate saturating graphs are planar embedded and edge 2-connected.

Property 4. Any edge of a candidate saturating graph has exactly one conflict with an edge of a different candidate saturating graph.

We now prove that each simplification performed by the algorithm preserves the possibility of finding a saturator of $C$. Consider simplification $s_{m}$, that is performed at a certain step of the simplification phase. $s_{m}$ can be one of Simplification 1, 2, or 3. Denote by $s_{0}, s_{1}, \ldots, s_{m-1}$ the simplifications performed before $s_{m}$ and denote by $E$ the set of edges collapsed while applying $s_{0}, s_{1}, \ldots, s_{m-1}$. Inductively, suppose that if an acyclic saturator of $C$ exists, there exists an acyclic saturator composed by the edges of $E$ plus some of the edges remaining in the $\mathcal{G}_{i}$ 's after simplifications $s_{0}, s_{1}, \ldots, s_{m-1}$. This is indeed the case when no simplification has been performed yet.

Lemma 4. Consider an edge ef $\mathcal{G}_{i}$ with no conflict. We have that $C$ admits a saturator only if it admits an acyclic saturator containing e and containing the edges of E.

Proof: Suppose $C$ admits a saturator. Then, by Lemma 1 and by inductive hypothesis, it admits an acyclic saturator $S$ such that $E \subseteq S$. If $S$ contains $e$ the statement follows. Otherwise, observe that since $S$ is a saturator, there exists a set $S^{\prime} \subseteq S$ of edges forming a path between the end-vertices $u$ and $v$ of $e$. Hence, the edges of $S^{\prime} \cup\{e\}$ form a cycle. Not all the edges of $S^{\prime}$ belong to $E$, otherwise $u$ and $v$ would not have been distinct vertices in $\mathcal{G}_{i}$ after simplifications $s_{0}, s_{1}, \ldots, s_{m-1}$. Hence, the set $S^{*}$ of edges obtained from $S$ by inserting $e$ and by removing any edge of $S^{\prime}$ not in $E$ is an acyclic saturator of $C$ containing $E$ and $e$. Namely, all the connected components of $C$ are connected by a path of edges in $S^{*}$ and since $e$ has no conflict and $S$ is a saturator, then no two edges in $S^{*}$ have a conflict. 
Lemma 5. Consider two edges $e_{i}$ and $e_{j}$ of two distinct multigraphs $\mathcal{G}_{i}$ for cluster $\mu_{i}$ and $\mathcal{G}_{j}$ for cluster $\mu_{j}$, respectively. Suppose that $e_{i}$ and $e_{j}$ conflict each other. Also, suppose that $e_{i}$ is a separating edge, while $e_{j}$ is not. Then $C$ admits a saturator only if it admits an acyclic saturator containing $e_{i}$, containing $E$, and not containing $e_{j}$.

Proof: Suppose $C$ admits a saturator. Then, by Lemma 1 and by inductive hypothesis, it admits an acyclic saturator $S$ such that $E \subseteq S$. Since at step $s_{m}$ end-vertices $u$ and $v$ of $e_{i}$ are in $\mathcal{G}_{i}$, then no path composed by edges of $E$ connects $u$ and $v$. Since $e_{i}$ is a separating edge, then if $e_{i}$ is not in $S$ adding the edges of $S$ to $G$ would not connect $G\left(\mu_{i}\right)$. Hence $e_{i} \in S$. Since no two conflicting edges can be in $S$, then $e_{j} \notin S$.

Lemma 6. Consider two separating edges $e_{i}$ and $e_{j}$ of two distinct multigraphs $\mathcal{G}_{i}$ for cluster $\mu_{i}$ and $\mathcal{G}_{j}$ for cluster $\mu_{j}$, respectively. Suppose that $e_{i}$ and $e_{j}$ conflict each other. We have that $C$ is not c-planar.

Proof: Suppose that $C$ admits a saturator. Then, by inductive hypothesis, it admits an acyclic saturator $S$ such that $E \subseteq S$. Since at step $s_{m}$ the end-vertices $u$ and $v$ of $e_{i}$ (the end-vertices $w$ and $x$ of $e_{j}$ ) are in $\mathcal{G}_{i}$ (are in $\mathcal{G}_{j}$ ), then no path composed by edges of $E$ connects $u$ and $v$ (connects $w$ and $x$ ). Since $e_{i}$ and $e_{j}$ are separating edges, then if $e_{i}\left(e_{j}\right)$ is not in $S$, adding the edges of $S$ to $G$ would not connect $G\left(\mu_{i}\right)\left(G\left(\mu_{j}\right)\right)$. However, $S$ cannot contain both $e_{i}$ and $e_{j}$, that conflict each other.

Let $\mu_{i}$ and $\mu_{j}$ be two distinct clusters admitting candidate saturating graphs $\mathcal{G}_{i}^{*}$ and $\mathcal{G}_{j}^{*}$, respectively. We define graph $\mathcal{G}_{i, j}^{*}$ as the planar embedded subgraph of $\mathcal{G}_{i}^{*}$ induced by the edges having a conflict with the edges of $\mathcal{G}_{j}^{*}$. We have:

Theorem 2. A single-conflict embedded flat clustered graph $C(G, T)$ is c-planar iff: (1) $G$ is planar; (2) There exists a face $f$ in $G$ such that when $f$ is chosen as outer face for $G$ no cycle composed by vertices of the same cluster encloses a vertex of a different cluster; (3) Each cluster of $C$ admits a candidate saturating graph; (4) For each pair of distinct clusters $\mu_{i}$ and $\mu_{j}, \mathcal{G}_{i, j}^{*}$ is edge 2-connected; and (5) For each pair of distinct clusters $\mu_{i}$ and $\mu_{j}, \mathcal{G}_{i, j}^{*}$ is dual to $\mathcal{G}_{j, i}^{*}$.

Proof: Let $S$ be an acyclic saturator of $C$ and let $u$ and $v$ be two vertices of candidate saturating graph $\mathcal{G}_{i}^{*}$. Denote by $S(u, v)$ the path of $S$ connecting $u$ and $v$. If edges $e_{i}$ and $e_{j}$ of candidate saturating graphs $\mathcal{G}_{i}^{*}$ and $\mathcal{G}_{j}^{*}$ conflict each other, we write $e_{i} \oplus e_{j}$.

The necessity of Conditions (1) and (2) descends from the one of Conditions 1 and 2 of Theorem 1 We prove the necessity of Condition (3). Suppose that $C$ does not admit candidate saturating graphs. Two cases are possible: Either before the simplification phase one of the $\mathcal{G}_{i}$ 's is not connected, or during the simplification phase two separating conflicting edges are found. In the former case the non- $c$-planarity of $C$ descends from Lemma 3 , in the latter case from Lemma 6 .

Now we deal with Condition (4). Suppose that $\mathcal{G}_{i, j}^{*}$ is not connected. Denote by $v_{1}$ and $v_{2}$ vertices in different connected components. Suppose, for a contradiction, that an acyclic saturator $S$ of $C$ exists. Consider $S\left(v_{1}, v_{2}\right)$ (see Fig. 2]a). Since $v_{1}$ and $v_{2}$ are in different components of $\mathcal{G}_{i, j}^{*}$, there exists an edge $(u, v) \in S\left(v_{1}, v_{2}\right)$ s. t. $(u, v) \oplus(w, x)$, where $(w, x) \in \mathcal{G}_{k}^{*}$, with $k \neq i, j$. Consider $S(w, x)$. Each edge of $S(w, x)$ cannot have a conflict with any edge of $S\left(v_{1}, v_{2}\right)$, otherwise $S$ would contain two conflicting edges, and with any edge $e$ of $\mathcal{G}_{i, j}^{*}$, otherwise $e$ would conflict with 
two candidate saturating edges. Hence, $\mathcal{G}_{j, i}^{*}$ has at least two connected components. Let $u_{1}$ and $u_{2}$ be two vertices in such components, respectively. Then, $S\left(u_{1}, u_{2}\right)$ either contains an edge $e_{1}$ s.t. $e_{1} \oplus e_{2}$, with $e_{2} \in S\left(v_{1}, v_{2}\right)$, or contains an edge $e_{1}$ s. t. $e_{1} \oplus e_{2}$, with $e_{2} \in S(w, x)$, implying that $S$ contains two conflicting edges.

Now suppose that $\mathcal{G}_{i, j}^{*}$ has a separating edge $(u, v)$. By construction $(u, v) \oplus(w, x)$, where $(w, x) \in \mathcal{G}_{j, i}^{*}$. Suppose, for a contradiction, that a saturator $S$ of $C$ exists.

1. If $(u, v) \notin S$, then consider $S(u, v)$ (see Fig. 2. b). Since $(u, v)$ is a separating edge for $\mathcal{G}_{i, j}^{*}$, then there exists an edge $\left(u^{\prime}, v^{\prime}\right) \in S(u, v)$ s. t. $\left(u^{\prime}, v^{\prime}\right) \oplus\left(w^{\prime}, x^{\prime}\right)$, where $\left(w^{\prime}, x^{\prime}\right) \in \mathcal{G}_{k}^{*}$, with $k \neq i, j$. Hence, $S\left(w^{\prime}, x^{\prime}\right)$ either contains an edge $e_{1} \mathrm{~s}$. t. $e_{1} \oplus e_{2}$, with $e_{2} \in S(u, v)$, implying that $S$ contains two conflicting edges, or contains an edge $e_{1}$ conflicting with $(u, v)$, implying that $(u, v)$ conflicts with two candidate saturating edges.

2. If $(u, v) \in S$, then consider $S(w, x)$.

- If an edge $\left(w^{\prime}, x^{\prime}\right) \in S(w, x)$ is s. t. $\left(w^{\prime}, x^{\prime}\right) \oplus\left(u^{\prime}, v^{\prime}\right)$, with $\left(u^{\prime}, v^{\prime}\right) \notin \mathcal{G}_{i, j}^{*}$, a contradiction is obtained as in the previous case (see Fig. 2. $\mathrm{c}$ ).

- Otherwise, consider any edge $\left(w^{\prime}, x^{\prime}\right) \in S(w, x)$ and edge $\left(u^{\prime}, v^{\prime}\right) \in \mathcal{G}_{i, j}^{*}$ s. t. $\left(u^{\prime}, v^{\prime}\right) \oplus\left(w^{\prime}, x^{\prime}\right)$. Let $v\left(v^{\prime}\right)$ be the endpoint of $(u, v)$ (resp. of $\left.\left(u^{\prime}, v^{\prime}\right)\right)$ outside cycle $S(w, x) \cup(w, x)$.

- If $u=u^{\prime}$ or if all edges of $S\left(u, u^{\prime}\right)$ have conflicts with edges of $\mathcal{G}_{j, i}^{*}$ (see Fig. 2]d), consider $S\left(v, v^{\prime}\right)$. Then there exists an edge $\left(u^{\prime \prime}, v^{\prime \prime}\right) \in S\left(v, v^{\prime}\right)$ s. t. $\left(u^{\prime \prime}, v^{\prime \prime}\right) \oplus\left(w^{\prime \prime}, x^{\prime \prime}\right)$, where $\left(w^{\prime \prime}, x^{\prime \prime}\right) \in \mathcal{G}_{k}^{*}$, with $k \neq i, j$, otherwise $(u, v)$ would not be a separating edge. Hence, $S\left(w^{\prime \prime}, x^{\prime \prime}\right)$ either contains an edge $e_{1}$ s. t. $e_{1} \oplus e_{2}$, with $e_{2} \in S\left(v, v^{\prime}\right)$, implying that $S$ contains two conflicting edges, or an edge $e_{1}$ s. t. $e_{1} \oplus e_{2}$, with $e_{2} \in S\left(u, u^{\prime}\right)$, implying that $S$ contains two conflicting edges, or an edge $e_{1}$ s. t. $e_{1} \oplus\left(u^{\prime}, v^{\prime}\right)$, implying that $\left(u^{\prime}, v^{\prime}\right)$ conflicts with two candidate saturating edges, or an edge $e_{1}$ s. t. $e_{1} \oplus(u, v)$, implying that $(u, v)$ conflicts with two candidate saturating edges.

- If $u \neq u^{\prime}$ and $S\left(u, u^{\prime}\right)$ contains at least one edge $\left(u^{\prime \prime}, v^{\prime \prime}\right)$ s. t. $\left(u^{\prime \prime}, v^{\prime \prime}\right) \oplus$ $\left(w^{\prime \prime}, x^{\prime \prime}\right)$, where $\left(w^{\prime \prime}, x^{\prime \prime}\right) \in \mathcal{G}_{k}^{*}$, with $k \neq i, j$ (see Fig.2. 2 e), then $S\left(w^{\prime \prime}, x^{\prime \prime}\right)$ contains either an edge $e_{1}$ s. t. $e_{1} \oplus e_{2}$, with $e_{2} \in S(w, x)$, implying that $S$ contains two conflicting edges, or an edge $e_{1}$ s. t. $e_{1} \oplus e_{2}$, with $e_{2} \in S\left(u, u^{\prime}\right)$, or an edge $e_{1}$ s. t. $e_{1} \oplus\left(u^{\prime}, v^{\prime}\right)$, implying that $\left(u^{\prime}, v^{\prime}\right)$ conflicts with two candidate saturating edges, or an edge $e_{1}$ s. t. $e_{1} \oplus(w, x)$, implying that $(w, x)$ conflicts with two candidate saturating edges.

Now we prove the necessity of Condition (5). Each edge of $\mathcal{G}_{i, j}^{*}$ has a conflict with (and hence is dual to) one edge of $\mathcal{G}_{j, i}^{*}$ and vice versa. By the necessity of Condition (4), we can assume that both $\mathcal{G}_{i, j}^{*}$ and $\mathcal{G}_{j, i}^{*}$ are edge 2 -connected. Hence $\mathcal{G}_{i, j}^{*}$ is not dual to $\mathcal{G}_{j, i}^{*}$ only if there is a face of $\mathcal{G}_{i, j}^{*}$ that contains in its interior two vertices of $\mathcal{G}_{j, i}^{*}$, or vice versa. Suppose w.l.o.g. that a face $f$ of $\mathcal{G}_{i, j}^{*}$ contains in its interior two vertices $u$ and $v$ of $\mathcal{G}_{j, i}^{*}$. Suppose, for a contradiction, that a saturator $S$ of $C$ exists. Consider $S(u, v)$.

1. If the vertices of $S(u, v)$ are in part inside $f$ and in part outside $f$ (see Fig. 2 $\mathrm{f}$ ), consider two vertices $v_{1}$ and $v_{2}$ in different connected components, disconnected by $S(u, v)$, of $f$. Consider $S\left(v_{1}, v_{2}\right)$. There exists an edge $(w, x) \in S\left(v_{1}, v_{2}\right)$ s. t. 


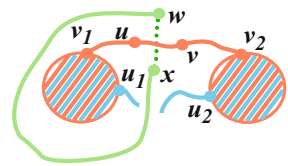

(a)

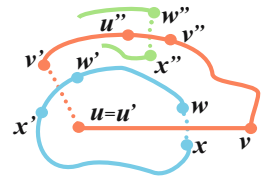

(d)

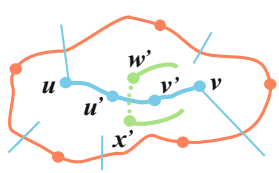

(g)

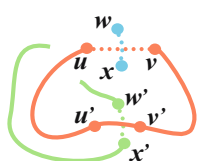

(b)

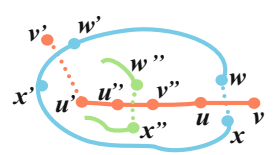

(e)

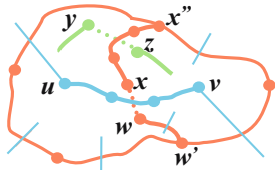

(h)

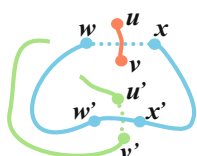

(c)

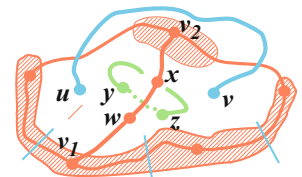

(f)

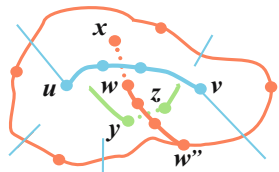

(i)

Fig. 2. Illustrations for the necessity of the conditions of Theorem 2 Edges of $\mathcal{G}_{i}^{*}$ are red, edges of $\mathcal{G}_{j}^{*}$ are light blue, and edges of $\mathcal{G}_{k}^{*}$ are green.

$(w, x) \oplus(y, z)$, where $(y, z) \in \mathcal{G}_{k}^{*}$, with $k \neq i, j$, otherwise $f$ would not be a face. Hence, $S(y, z)$ either contains an edge $e_{1}$ s. t. $e_{1} \oplus e_{2}$, with $e_{2} \in S\left(v_{1}, v_{2}\right)$, implying that $S$ contains two conflicting edges, or an edge $e_{1}$ conflicting with an edge $e_{2} \in f$, implying that $e_{2}$ conflicts with two candidate saturating edges.

2. Otherwise, $S(u, v)$ is composed by vertices all lying inside $f$.

- If there is an edge $\left(u^{\prime}, v^{\prime}\right) \in S(u, v)$ s. t. $\left(u^{\prime}, v^{\prime}\right) \oplus\left(w^{\prime}, x^{\prime}\right)$, where $\left(w^{\prime}, x^{\prime}\right) \in$ $\mathcal{G}_{k}^{*}$, with $k \neq i, j$ (see Fig. 2 g), then $S\left(w^{\prime}, x^{\prime}\right)$ either contains an edge $e_{1}$ s. t. $e_{1} \oplus e_{2}$, with $e_{2} \in S(u, v)$, implying that $S$ contains two conflicting edges, or an edge $e_{1}$ s. t. $e_{1} \oplus e_{2}$, with $e_{2} \in f$, implying that $e_{2}$ conflicts with two candidate saturating edges, or an edge $e_{1}$ s. t. $e_{1} \oplus e_{2}$, with $e_{2}$ dual to an edge of $f$, implying that $e_{2}$ conflicts with two candidate saturating edges.

- Otherwise, any edge of $S(u, v)$ is dual to an edge of $\mathcal{G}_{i, j}^{*}$. Consider any edge $(w, x)$ dual to an edge of $S(u, v)$.

- If $w \in f$ or if there exists a vertex $w^{\prime} \in f$ s. t. any edge of $S\left(w, w^{\prime}\right)$ conflicts with an edge of $\mathcal{G}_{j, i}^{*}$ (see Fig. 2] h), then $x \notin f$ and there exists no vertex $x^{\prime}$ in $f$ s. t. all edges of $S\left(x, x^{\prime}\right)$ conflict with edges of $\mathcal{G}_{j, i}^{*}$, otherwise $f$ would not be a face. Consider any vertex $x^{\prime \prime} \in f$ and $S\left(x, x^{\prime \prime}\right)$. Then, there exists an edge in $S\left(x, x^{\prime \prime}\right)$ that has a conflict with an edge $(y, z)$ in $\mathcal{G}_{k}^{*}$, with $k \neq i, j$. Hence, $S(y, z)$ either contains an edge $e_{1}$ s. t. $e_{1} \oplus e_{2}$, with $e_{2} \in S(u, v)$, implying that $S$ contains two conflicting edges, or contains an edge $e_{1}$ s. t. $e_{1} \oplus e_{2}$, with $e_{2} \in S\left(x, x^{\prime \prime}\right)$, implying that $S$ contains two conflicting edges, or contains an edge $e_{1}$ s. t. $e_{1} \oplus e_{2}$, with $e_{2} \in f$, implying that $e_{2}$ conflicts with two candidate saturating edges, or contains an edge $e_{1}$ s. t. $e_{1} \oplus e_{2}$, with $e_{2}$ dual to an edge in $f$, implying that $e_{2}$ conflicts with two candidate saturating edges. 
- If $w \notin f$ and there exists no vertex $w^{\prime} \in f$ s. t. any edge of $S\left(w, w^{\prime}\right)$ conflicts with an edge of $\mathcal{G}_{j, i}^{*}$ (see Fig. 2 $\mathrm{i}$ ), then there exists a vertex $w^{\prime \prime} \in$ $f$ s. t. $S\left(w, w^{\prime \prime}\right)$ contains an edge $e_{1}$ s. t. $e_{1} \oplus(y, z)$, with $(y, z) \in \mathcal{G}_{k}^{*}$, with $k \neq i, j$, and a contradiction is derived as in the previous case.

We prove the sufficiency of Conditions $1,2,3,4$, and 5 for the $c$-planarity of $C$. Consider any planar drawing of $G$ satisfying Conditions 1 and 2 and hence satisfying Conditions 1 and 2 of Theorem 1 . We show how to construct an acyclic saturator $S$ of $C$ satisfying Condition 3 of Theorem 1. Apply the simplification phase, choosing an acyclic set $E$ of edges to be in $S$ and obtaining a candidate saturating graph $\mathcal{G}_{i}^{*}$ for each cluster $\mu_{i}$ (this can be done since $C$ satisfies Condition 3 ). Order the clusters in whichever way $\mu_{1}, \mu_{2}, \ldots, \mu_{m}$. For any pair of clusters $\mu_{i}$ and $\mu_{j}$, with $i<j$, choose a spanning tree $\mathcal{T}_{i, j}^{*}$ of $\mathcal{G}_{i, j}^{*}\left(\mathcal{T}_{i, j}^{*}\right.$ can be found since, by Condition $4, \mathcal{G}_{i, j}^{*}$ is edge 2 connected). Remove from $\mathcal{G}_{j, i}^{*}$ all edges dual to edges of $\mathcal{T}_{i, j}{ }^{*}$, obtaining a graph $\mathcal{T}_{j, i}^{*}$. We claim that $\mathcal{T}_{j, i}^{*}$ is a spanning tree of $\mathcal{G}_{j, i}^{*}$. By Condition $5, \mathcal{G}_{i, j}^{*}$ and $\mathcal{G}_{j, i}^{*}$ are dual graphs, and the edges of a cycle in $\mathcal{G}_{i, j}^{*}$ are dual to the edges of a cutset in $\mathcal{G}_{j, i}^{*}$, and vice versa (Lemma 1.4 of [11]). Hence, if $\mathcal{T}_{j, i}^{*}$ has more than one connected component, the edges removed from $\mathcal{G}_{j, i}^{*}$ form a cutset for $\mathcal{G}_{j, i}^{*}$, and those of $\mathcal{T}_{i, j}{ }^{*}$ form a cycle, contradicting the hypothesis that $\mathcal{T}_{i, j}^{*}$ is a tree. If a set of edges of $\mathcal{T}_{j, i}^{*}$ is a cycle, the edges dual to such a cycle form a cutset for $\mathcal{G}_{i, j}^{*}$, contradicting the hypothesis that $\mathcal{T}_{i, j}^{*}$ is spanning for $\mathcal{G}_{i, j}^{*}$. For any pair of clusters $\mu_{i}$ and $\mu_{j}$, with $i<j$, add the edges of $\mathcal{T}_{i, j}^{*}$ and of $\mathcal{T}_{j, i}^{*}$ to $S$. We claim that $S$ is a saturator of $C$. Edges chosen in the simplification phase do not conflict each other by construction. Such edges do not conflict with edges of trees $\mathcal{T}_{i, j}^{*}$. In fact, an edge in $\mathcal{T}_{i, j}^{*}$ conflicts only with an edge in $\mathcal{G}_{j}^{*}$, with $i \neq j$. By construction, edges of the $\mathcal{T}_{i, j}{ }^{*}$ 's do not conflict each other. Hence, $S$ does not have two conflicting edges. It's easy to see that, after $G$ has been augmented to a graph $G^{\prime}$ by adding the edges of $S$ to it, each cluster $\mu_{i}$ has exactly one connected component. Namely, distinct connected components of $G\left(\mu_{i}\right)$ are represented after the simplification phase by distinct vertices in $\mathcal{G}_{i}^{*}$, that is edge 2 -connected and that is partitioned in edge 2-connected subgraphs $\mathcal{G}_{i, j}^{*}$. Since a spanning tree is chosen to be in $S$ for any $\mathcal{G}_{i, j}^{*}$, then $\bigcup_{j} \mathcal{T}_{i, j}^{*}$ is spanning for $\mathcal{G}_{i}^{*}$ and $G^{\prime}\left(\mu_{i}\right)$ has exactly one connected component. Finally, suppose that $G^{\prime}\left(\mu_{i}\right)$ has a cycle $c$ containing an edge of $S$. By construction, edges chosen in the simplification phase only join different connected components of $G\left(\mu_{i}\right)$ and no edge of $c$ could belong to some $\mathcal{G}_{i, j}^{*}$, otherwise $G^{\prime}\left(\mu_{j}\right)$ would be disconnected.

Theorem 3. An embedded flat clustered graph $C(G, T)$ with at most five vertices per face is c-planar if and only if: (1) $G$ is planar; (2) There exists a face $f$ in $G$ such that when $f$ is chosen as outer face for $G$ no cycle composed by vertices of the same cluster encloses a vertex of a different cluster; (3) Each cluster of $C$ admits a candidate saturating graph; and (4) For each pair of distinct clusters $\mu_{i}$ and $\mu_{j}, \mathcal{G}_{i, j}^{*}$ is edge 2-connected; and (5) For each pair of distinct clusters $\mu_{i}$ and $\mu_{j}, \mathcal{G}_{i, j}^{*}$ is dual to $\mathcal{G}_{j, i}^{*}$.

Proof: Consider any face $f$ of $G$. Since $f$ has at most five vertices, then it has at most two connected components of each cluster, so it has at most one candidate saturating edge per cluster. Since at least two vertices are necessary for each candidate saturating edge, then $f$ contains candidate saturating edges for at most two clusters. Hence, $C$ is a single-conflict embedded flat clustered graph and Theorem 2 applies. 


\section{An Efficient $c$-Planarity Testing Algorithm}

We use Theorem 3 to derive a linear time and space $c$-planarity testing algorithm for embedded flat clustered graphs with at most five vertices per face. The algorithm can be extended to test the $c$-planarity of single-conflict embedded flat clustered graphs relying on Theorem 2. The details of the extension are omitted for brevity. Anyway, we will emphasize the steps of the algorithm that have to be modified for this purpose.

Let $C(G, T)$ be an $n$-vertex embedded flat clustered graph with at most five vertices per face. To test Condition (1) of Theorem 3 , it is sufficient to test if $G$ is a planar embedding. This can be done in $O(n)$ time and space with the techniques in [10].

To test Condition (2), we observe that a face exists satisfying such a condition iff the embedded clustered graph is hole-free, that is, chosen an arbitrary face as external, a cycle $c$ of $G$ doesn't exist composed by vertices of the same cluster $\mu$ such that $c$ has a vertex inside and a vertex outside both belonging to clusters different from $\mu$. A linear-time algorithm for checking if an embedded clustered graph is hole-free has been provided in [5] in the case of $c$-connected clustered graphs. However, we can use the same algorithm because of the following lemma.

Lemma 7. Let $C(G, T)$ be a clustered graph. Let $C^{\prime}\left(G, T^{\prime}\right)$ be the c-connected clustered graph obtained from $C$ as follows. Each node $\nu$ of $T$ is replaced in $T^{\prime}$ by nodes $\nu_{1}, \ldots, \nu_{h}$, one for each of the $h \geq 1$ connected components of $G(\nu)$. Let $\mu_{1}, \ldots, \mu_{k}$ be the nodes replacing the parent $\mu$ of $\nu$. The parent of $\nu_{j}$ in $T^{\prime}$ is the node $\mu_{i}$ such that $G\left(\nu_{j}\right)$ is a subgraph of $G\left(\mu_{i}\right)$. We have that $C$ is hole-free iff $C^{\prime}$ is hole-free.

In order to test Condition (3) we create multigraphs $\mathcal{G}_{i}$. This is done in $O(n)$ time as follows. Connected Components. For each node $\mu$ of $T$ compute the connected components of $G(\mu)$. This is done in linear time and space. Candidate saturating edges. We insert candidate saturating edges inside the faces of $G$. Consider a face $f$. Construct maximal sequences of vertices consecutive on the border of $f$ and belonging to the same cluster. For any two sequences $S_{1}$ and $S_{2}$ that have vertices belonging to the same cluster, take a vertex $v_{1} \in S_{1}$ and a vertex $v_{2} \in S_{2}$. If the connected component associated to $v_{1}$ is different from the one associated to $v_{2}$ (this can be tested in constant time), then insert a candidate saturating edge between $v_{1}$ and $v_{2}$. At most two edges are inserted inside $f$. The described insertion can be performed in constant time and hence in linear time for all faces of $G$. This step is more tricky when considering singleconflict clustered graphs. In this case, in order to achieve total linear time special care must be put when considering groups of candidate saturating edges between vertices of the same cluster and when determining the conflicts between candidate saturating edges. Multigraphs $\mathcal{G}_{\mathbf{i}}$. Consider cluster $\mu_{i}$. Add a vertex to $\mathcal{G}_{i}$ for each connected component of $G\left(\mu_{i}\right)$. For each candidate saturating edge $e$ insert an edge between the connected components joined by $e$. The construction of the $\mathcal{G}_{i}$ 's can be done so that their embeddings are those induced by the adjacencies of the faces of $G$. Further, such a construction can be done in linear time and space because of the following:

Property 5. $\sum_{\mu_{i}}\left|\mathcal{G}_{i}\right|=O(n)$, where $\left|\mathcal{G}_{i}\right|$ is the size of the graph.

Property [5] does not hold when considering single-conflict embedded flat clustered graphs, that can generally have faces with a linear number of incident vertices. However, 
the arrangement of the candidate saturating edges in the single-conflict setting allows to reduce the size of the construction introducing only an overall linear number of them.

Now we show how to test if Condition (3) of Theorem 3 is satisfied. First, test if the $\mathcal{G}_{i}$ 's are connected. If not, return non-c-planar.

We equip each $\mathcal{G}_{i}$ with a data structure supporting the following update and query operations: remove an edge, collapse (identify the end-vertices of) an edge and merge the embeddings of its end-vertices, answer if an edge is a separating edge, answer if an edge has a conflict and in case output the conflicting edge. Observe the difference between the above definition of the collapse operation and the one given in Section 3 . A data structure exists that can be set-up in linear time and that performs each of the above operations in constant time. In fact, all of them are trivial graph operations, with the exception of answering if an edge $e$ is a separating edge. We equip each edge with two pointers to the two identifiers of the incident faces. When an edge $e$ is removed from $\mathcal{G}_{i}$ we simply modify the identifier of one of the two faces former incident on $e$. To answer the query in constant time we check if the two faces around $e$ are the same face. Also, we compute a set $\mathcal{F}$ of candidate saturating edges that have no conflict. For each edge $e$ of $\mathcal{F}$ we compute the set $\mathcal{E}$ of edges parallel to $e$. Such computations are performed in linear time. We will show how to use $\mathcal{F}$ and sets $\mathcal{E}$ during the simplification steps.

We show how to apply Simplification 1. Construct the set $\mathcal{F}^{\prime}$ of the edges of any spanning forest of $\mathcal{F}$. Set $\mathcal{F}^{\prime \prime}=\emptyset$. Take each edge $e_{1}$ of $\mathcal{F}^{\prime}$. Consider the set $\mathcal{E}$ of edges parallel to $e_{1}$. For each edge $e_{2} \neq e_{1}$ in $\mathcal{E}$, if $e_{2}$ has a conflict with an edge $e_{2}^{*}$, add $e_{2}^{*}$ to $\mathcal{F}^{\prime \prime}$. After this work has been performed on all the edges of $\mathcal{F}^{\prime}$, collapse all of such edges, removing self-loops. Set $\mathcal{F}^{\prime \prime}$ contains all the edges that became conflict-free after the previous step. The edges of $\mathcal{F}^{\prime \prime}$ do not have multiple edges:

\section{Lemma 8. The edges of set $\mathcal{F}^{\prime \prime}$ do not have multiple edges.}

Perform Simplification 1 on the edges of $\mathcal{F}^{\prime \prime}$. The above lemma guarantees that after this second pass no new conflict-free edge can be originated.

Now, Simplification 2 is applied till the $\mathcal{G}_{i}$ 's are edge 2 -connected or the non- $c$ planarity of $C$ is stated. First, construct a set $\mathcal{H}$ of separating edges as follows. For each edge $e$ in $\mathcal{G}_{i}$ verify if the faces incident to $e$ are the same. If yes, then add $e$ to $\mathcal{H}$. This computation takes time linear in the number of edges in the $\mathcal{G}_{i}$ 's. Now, for each edge $e$ in $\mathcal{H}$, check if edge $e^{*}$ conflicting with $e$ belongs to $\mathcal{H}$. If yes, return non- $c$-planar, otherwise delete $e^{*}$ and collapse $e$. After this has been done for all edges in $\mathcal{H}$, other separating edges could have been created in $\mathcal{G}_{i}$. However, if this happens, then we can conclude that $C$ is not $c$-planar as stated in the following lemmas:

Lemma 9. Consider a face $f$ of $\mathcal{G}_{i}$. Suppose that $f$ contains a separating pair composed by edges $\left(u_{1}, u_{2}\right)$ and $\left(u_{3}, u_{4}\right)$. Suppose that $\left(u_{1}, u_{2}\right)$ has a conflict with edge $\left(v_{1}, v_{2}\right)$ that is a separating edge, and that $\left(u_{3}, u_{4}\right)$ has a conflict with edge $\left(v_{3}, v_{4}\right)$. We have that $C$ is not c-planar.

Lemma 10. Suppose that each edge of $\mathcal{H}$ has a conflict with a non-separating edge. Collapse the edges in $\mathcal{H}$, repeatedly applying Simplification 2. Either the resulting multigraphs $\mathcal{G}_{i}$ are edge 2-connected or $C$ is not c-planar. 
For each pair of distinct clusters $\mu_{i}$ and $\mu_{j}$, we check if $\mathcal{G}_{i, j}^{*}$ is edge 2 -connected (Condition (4) of Theorem 3) and if $\mathcal{G}_{i, j}^{*}$ is dual to $\mathcal{G}_{j, i}^{*}$ (Condition (5) of Theorem 3). This is easily done in linear time because of the following property.

Property 6. $\sum\left|\mathcal{G}_{i, j}^{*}\right|=O(n)$, where $\left|\mathcal{G}_{i, j}^{*}\right|$ is the size of the graph.

Hence, we can conclude the section with the following theorem.

Theorem 4. The c-planarity of an n-vertex embedded flat clustered graph $C(G, T)$ with at most five vertices per face can be tested in $O(n)$ time and space.

\section{Conclusions}

We remark that the simplification phase described in Section 3 is a preprocessing that can be performed on any embedded flat clustered graph. This allows to reduce the problem of testing the $c$-planarity of such graphs to the one of deciding whether a set of edge 2 -connected candidate saturating graphs admits a set of non-conflicting spanning trees. However, it's rather easy to see that the characterization shown in Theorem 2 does not hold for general embedded flat clustered graphs.

We conclude by providing a list of families of embedded clustered graphs for which, in our opinion, determining the time complexity of a $c$-planarity testing is worth of interest: (i) single-conflict general (non-flat) embedded clustered graphs; (ii) embedded flat clustered graphs where each face of the underlying graph has at most two (or a constant number of) vertices of the same cluster; and (iii) embedded flat clustered graphs.

\section{References}

1. Cornelsen, S., Wagner, D.: Completely connected clustered graphs. Journal of Discrete Algorithms 4(2), 313-323 (2006)

2. Cortese, P.F., Di Battista, G.: Clustered planarity. In: ACM SoCG 2005, pp. 32-34 (2005)

3. Cortese, P.F., Di Battista, G., Patrignani, M., Pizzonia, M.: Clustering cycles into cycles of clusters. Journal of Graph Algorithms and Applications 9(3), 391-413 (2005)

4. Cortese, P.F., Di Battista, G., Patrignani, M., Pizzonia, M.: On embedding a cycle in a plane graph. In: Healy, P., Nikolov, N.S. (eds.) GD 2005. LNCS, vol. 3843, pp. 49-60. Springer, Heidelberg (2006)

5. Dahlhaus, E.: A linear time algorithm to recognize clustered graphs and its parallelization. In: Lucchesi, C.L., Moura, A.V. (eds.) LATIN 1998. LNCS, vol. 1380, pp. 239-248. Springer, Heidelberg (1998)

6. Di Battista, G., Frati, F.: Efficient c-planarity testing for embedded flat clustered graphs with small faces. Tech. Report RT-DIA-119-2007, Dip. Inf. e Aut., Univ. Roma Tre (2007)

7. Feng, Q., Cohen, R.F., Eades, P.: Planarity for clustered graphs. In: Spirakis, P.G. (ed.) ESA 1995. LNCS, vol. 979, pp. 213-226. Springer, Heidelberg (1995)

8. Goodrich, M.T., Lueker, G.S., Sun, J.Z.: C-planarity of extrovert clustered graphs. In: Healy, P., Nikolov, N.S. (eds.) GD 2005. LNCS, vol. 3843, pp. 211-222. Springer, Heidelberg (2006)

9. Gutwenger, C., Jünger, M., Leipert, S., Mutzel, P., Percan, M., Weiskircher, R.: Advances in c-planarity testing of clustered graphs. In: Goodrich, M.T., Kobourov, S.G. (eds.) GD 2002. LNCS, vol. 2528, pp. 220-235. Springer, Heidelberg (2002)

10. Kirkpatrick, D.G.: Establishing order in planar subdivisions. Discrete \& Computational Geometry 3, 267-280 (1988)

11. Nishizeki, T., Chiba, N.: Planar Graphs: Theory and Algorithms. North-Holland (1988) 